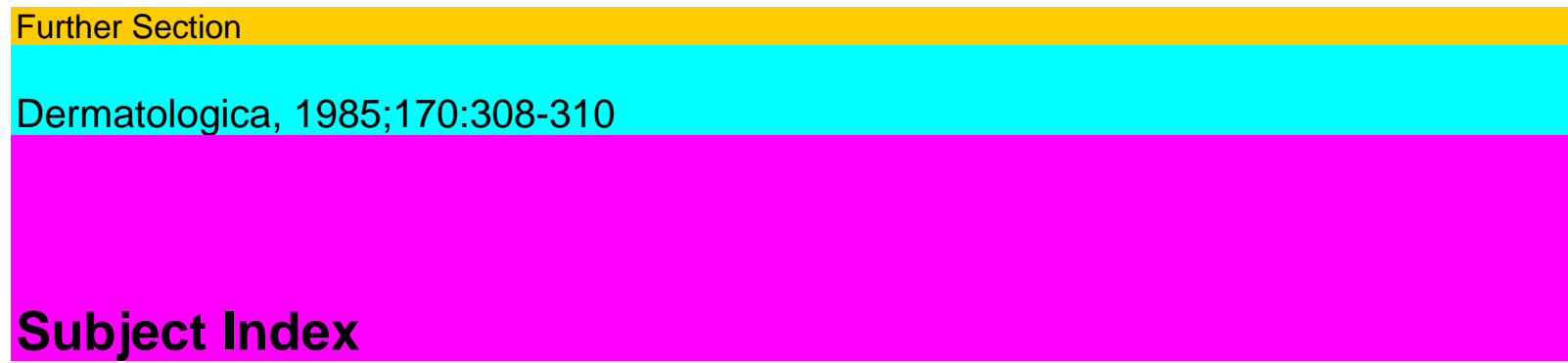

\title{
Acne 165
}

Acquired hypertrichosis lanuginosa 207

Adhesive 260

Adipose tissue 101

Alopecia areata 40, 69

American leishmaniasis 22

Angiosarcoma 286

Antilipoprotein IgGк 157

Argon laser 225

Arthritis 276

Atopic cataract 180

- dermatitis 35, 114, 180

Autoantibodies 80

Autoimmune diseases 86

Autoradiography 48

Basal cell nevus 293 Behçet's disease 265 Benzoylperoxide 165 Betamethasone valerate 195

Bindi 260 Biomechanics 105 Blanching 12 Blister 48 Breast cancer 170

- $\quad$ carcinoma 286

Bullous pemphigoid 86

Cancer 147 Candida 271 Carprofen 74

Cartilaginous metaplasia 249 Cell renewal 48 Cephalosporins 17 Cervix carcinoma 199

Chancroid 136 Chancroidal ulcer 136 Cheilitis glandularis 94 Chlamydia 77 Chronic urticaria 90

Cicatricial pemphigoid 256 Collagen 105

Contact allergen therapy 40

depigmentation 260 Corticosteroids 12 Cosmetic 260 Cutaneous leiomyoma 98 Cutaneous marker 128

pathergy test 265

Decreased lymphoproliferative response to mitogens

27 Dermatomyositis 6 Direct immunofluorescence 84 Discoid lupus erythematosus 263

Distribution pattern 235 Domoprednate 189, 195 Doxepin 90 jD-Penicillamine 297 Dyshidrosis

84

Eccrine porocarcinoma 202

- poroma 202

Epidemiology 35

Epidermodysplasia verruciformis 53, 213 Epidermolysis bullosa dystrophica 221 Epithelioid 43

Erythroderma 147 Etretinate 244 Exfoliative dermatitis 147

Familial epidermodysplasia verruciformis 53 Flow cytometry 1 Follicular mucinosis 133

Footwear 260 
Glycosaminoglycans 6 Gonoblennorrhoea adultorum 17 Gonococcal conjunctivitis 17 Gonococcemia 276

Haemophilus ducreyi 136 Hemangiomas 225 Hepatic immunopathy 31

Subject Index

309

Herpes simplex 65 Histopathology 105 HLA typing 53 HPV-1 213 HPV-3 213 HPV-16 199

Hyperpigmentation 225

Ichthyosis $180 \operatorname{IgA}$ myeloma $240 \operatorname{IgE} 114$

Immune complexes 80 Imniunosuppression 142 Inactivated measles virus 280 Induced

pemphigus 297 Interdigitating reticulum cells 121 Internal malignancy 147 Intestinal

immunopathy 31 Intradermal injection 302

Jaw cysts 293

Keratitis 17 Keratoacanthoma 221

- $\quad$ centrifugum marginatum 221

Killing of defective neutrophil leucocytes 27

Leiomyosarcoma 256

Lens 69

Lepromatous leprosy 80

Leukoderma 260

Lichen nitidus 253

Lipodystrophy, partial 101

Local anaesthesia 230

Localized pemphigoid 84

Low-grade squamous cell carcinoma 221

Lymphadenosis benigna cutis 121

Lymphangiosarcoma 286

Lymphedema 286

Lymphocyte blastogenesis 22

- $\quad$ transformation test 22

Male nipple 98 Malignant melanoma 48 Measles 280 Melanic pigment 225 Merkel cell

carcinoma 1 Metastatic malignant melanoma 249 Micropapular form 253 Monoclonal antibodies 59,121

Montenegro reaction 22 Multiple myeloma 157

sclerosis 86 Munchausen syndrome 94

-, bleeding type 94 Mycosis fungoides 59 Myotonic dystrophy 128

Nails 142

Natural measles virus 280

Nevoid basal cell epithelioma 293

Nodulocystic acne, treatment 185

Normolipemic plane xanthomatosis 157

Norwegian scabies 142

Nylon-clothes friction dermatosis 235

Oil bleomycin 302 Onychomycosis 142 Optoelectronic sensor 12 Oral Raynaud's phenomenon 263

Paget's disease of the nipple 170 
Papilloma virus genomes 199

Papillon-Lefèvre syndrome 27

Paraneoplastic syndrome 147

Patch test 260

Photoallergy 74

Photodermatitis 74

Photosensitivity 133

Phototoxicity 74

Phytohemagglutinin 22

Pilomatricoma 128

Pin pricking 230

Piroxicam 297

Placebo 189

PMNL chemotaxis 265

Prognosis 202

Progressive facial hemiatrophy 145

Pseudoepitheliomatous hyperplasia 221

Psoriasis 65, 195

in children 244

vulgaris 256 Pustular psoriasis 244

rash 280 Pustules 276

Rapid depletion of PMNL 265 Regressing plane warts 213 Reiter's disease 77 Respiratory atopy 180

310

Subject Index

Retinal pigmentary epithelium 69

13-c/s-Retinoic acid, low-dose treatment 185

Rheumatoid arthritis 297

Roaccutan and tetracycline, combined treatment 1

Romberg's syndrome 145

Rupioid psoriasis 77

Sarcoidosis 253

Sarcoma 43

Scabies 142

Scleroderma 105

Scléroderme en coup de sabre 145

Sebum excretion 165

Self-inflicted cheilorrhagia 94

Serum IgE 180

Skeletal defects 293

Skin atrophy 189

colonization 35

diseases 65

immunopathy 31

reflectance 12 
testing 114

window technique 265 Sneddon-Wilkinson disease 240 Soft tissue 43

Specific cell-mediated immunity 213 Squamous cell carcinoma 302 Squaric acid dibutyl ester 40 85 Staphylococcus aureus 35, 114 Stewart-Treves syndrome 286 Subcorneal pustular dermatosis 240 Synovial 43 Systemic lupus erythematosus 80, 263

T lymphocytes 59 Telangiectasia 189 Topical corticosteroids 189 - steroids 195 Tumour heterogeneity 1 Type- 1 diabetes 65

Ulcers 271

Unusual pigmentation 235

Urticaria 133

U V erythema 12

Vasoconstriction 12 Verrucous carcinoma 221 Viral antigens 213 Vitiligo 65 